\title{
Uric acid excretion in the relatives of patients with gout
}

\author{
J. T. SCOTT AND A. C. POLLARD
}

From the Clinical Division, Kennedy Institute of Rheumatology, and Charing Cross Hospital Medical School, London

This paper describes a study in which uric acid excretion in patients with gout was compared with that of their close relatives.

\section{Methods}

The patients all had a diagnosis of primary gout, i.e. they had a characteristic history of acute episodic arthritis with a serum uric acid level above $6 \mathrm{mg}$. $/ 100 \mathrm{ml}$. and no evident underlying cause for hyperuricaemia. The investigation was carried out before treatment with uratelowering drugs was started. The nature of the study was explained to them and to as many as possible of their adult first-degree relatives, and all the participants were carefully instructed in the details of a low-purine alcoholfree diet (National Institute of Health, Bethesda low purine diet: daily purine content approximately $200 \mathrm{mg}$.) which they took for 6 days. On the last day they collected a 24-hour urine sample in a provided container which they delivered within a few hours of completion, when a blood sample was also taken. Uric acid and creatinine levels in these samples were estimated by Technicon Auto-Analyzer.

46 patients with 107 adult relatives participated in the investigation. Of these patients, 33 had male relatives and 31 had female relatives, and figures from all these subjects were taken in the analysis relating to serum uric acid

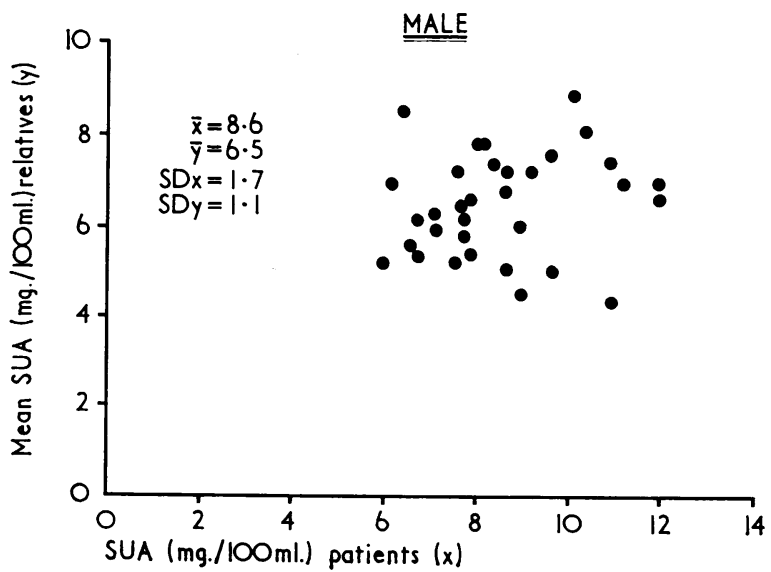

FIG. 1 Serum uric acid of patients plotted against mean serum uric acid of male relatives.

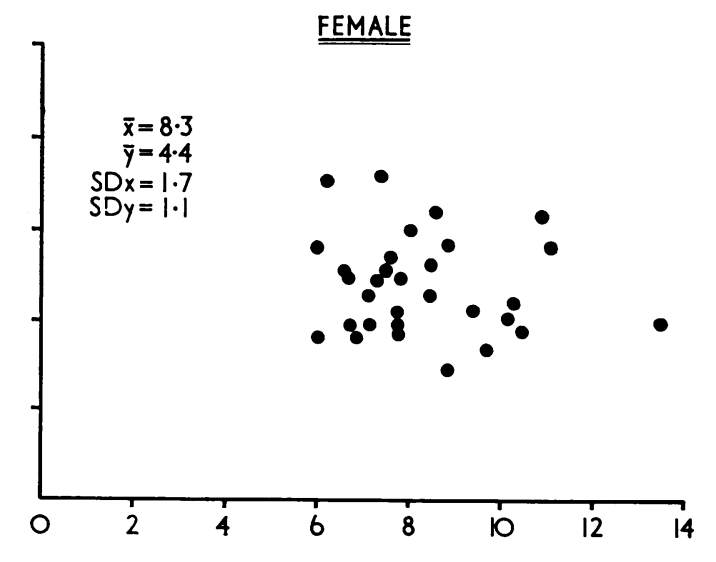

levels. Some patients and relatives were excluded from studies involving urine collections, either because creatinine clearance was less than $60 \mathrm{ml} . / \mathrm{min}$. (indicating either renal impairment or incomplete urine collection) or, in one instance, because dietary restrictions had clearly been disregarded. The final analysis relating to uric acid excretion therefore applied to 37 patients (36 men and 1 woman) and 96 relatives, of whom 42 were brothers, 40 sisters, 5 mothers, 3 fathers, 3 daughters, and 3 sons; 27 of the gout patients had male relatives and 26 had female relatives. Two of the relatives themselves had gout, but had not taken any treatment for several months.

\section{Results}

These are shown in Figs 1 to 8, where values for the patients are plotted along the horizontal axes and mean values for the corresponding relatives along the vertical. Separate calculations are shown for male and female relatives.

\section{(1) Serum uric acid (Figs 1 and 2)}

The mean serum uric acid level in gout patients with male relatives was $8.6 \mathrm{mg}$. $/ 100 \mathrm{ml}$. (standard deviation (S.D.) 1.7) and in those with female relatives

FIG. 2 Serum uric acid of patients plotted against mean serum uric acid of female relatives. 

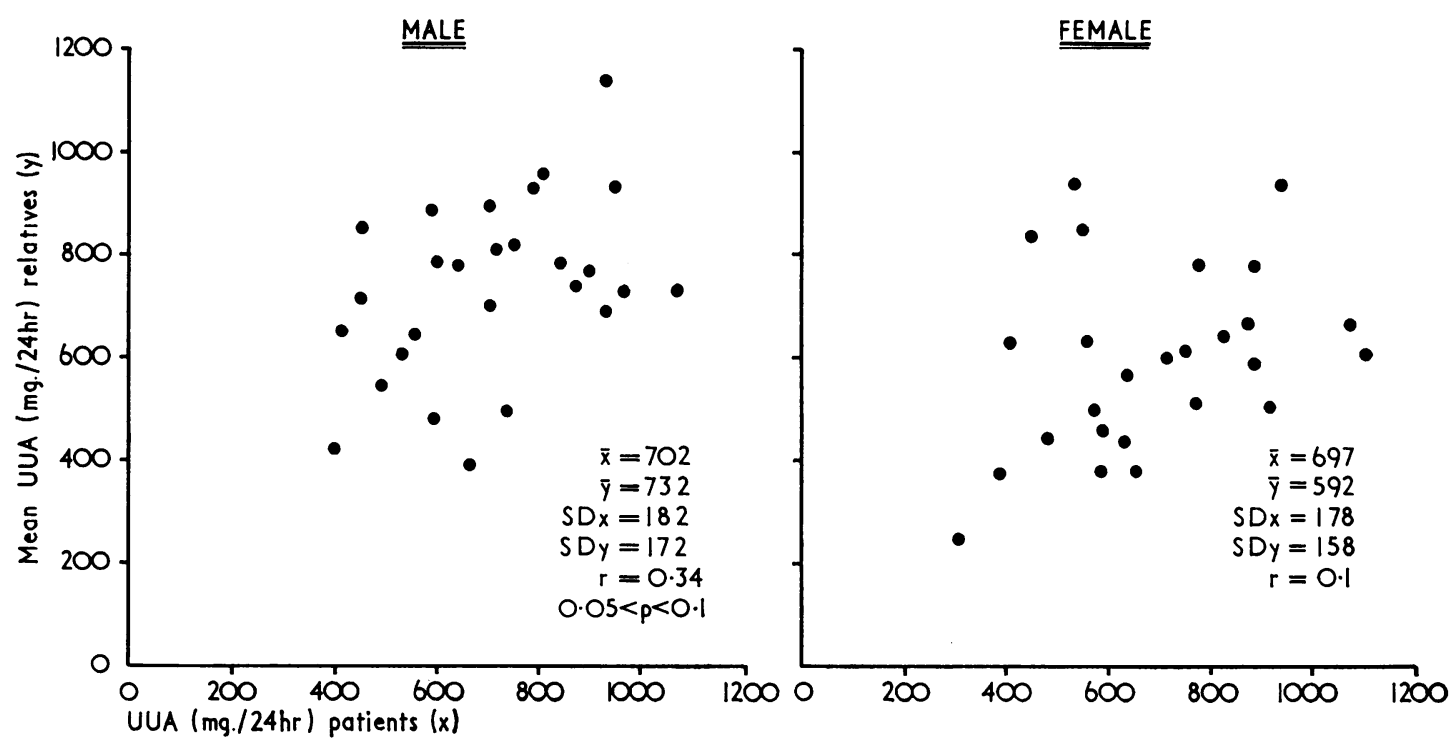

FIG. 3 24-hr urinary uric acid of patients plotted against mean 24-hr urinary uric acid of male relatives.

$8 \cdot 3 \mathrm{mg} . / 100 \mathrm{ml}$. (S.D. 1 · 7). Mean levels for male and female relatives were $6.5 \mathrm{mg} . / 100 \mathrm{ml}$. (S.D. 1 1 ) and $4 \cdot 4 \mathrm{mg} . / 100 \mathrm{ml}$. (S.D. 1.1) respectively. No correlation was apparent between uric acid levels in the patients and the mean levels of their relatives.

(2) 24-hr urinary uric acid (Figs 3 and 4).

The mean urinary uric acid level in gout patients with male relatives was $702 \mathrm{mg} . / 24 \mathrm{hrs}$ (S.D. 182), and in those with female relatives $697 \mathrm{mg} . / 24 \mathrm{hrs}$ (S.D. 178). Mean levels for male and female relatives were $732 \mathrm{mg} . / 24 \mathrm{hrs}$ (S.D. 172) and $592 \mathrm{mg} . / 24 \mathrm{hrs}$

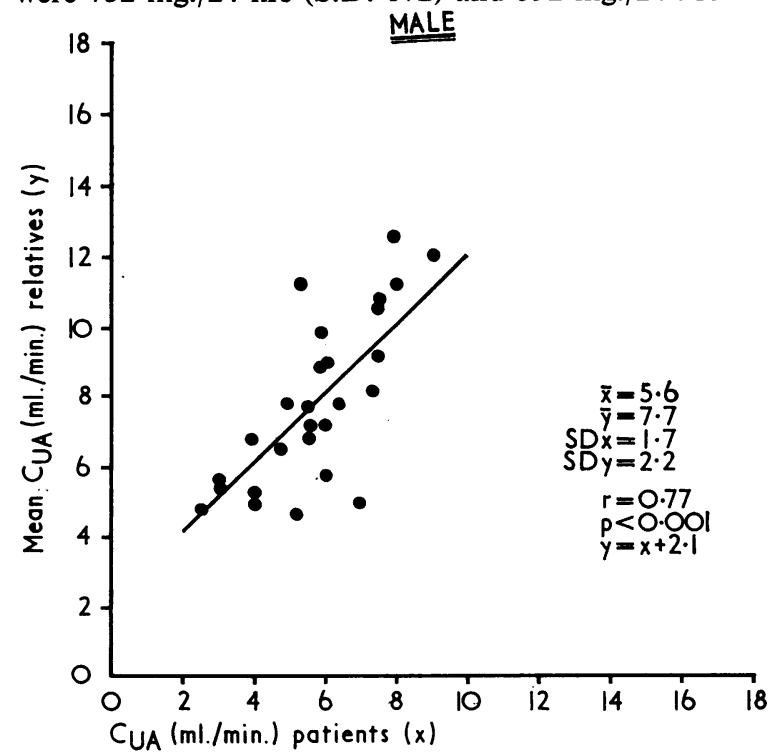

FIG. 4 24-hr urinary uric acid of patients plotted against mean 24-hr urinary uric acid of female relatives.

(S.D. 158) respectively.

Figs 3 and 4 show a possible tendency for higher values in the patients to be reflected in the mean values of their relatives, especially with regard to male relatives, but this does not achieve statistical significarce.

(3) Uric acid clearance (Figs 5 and 6)

This was obtained by calculating

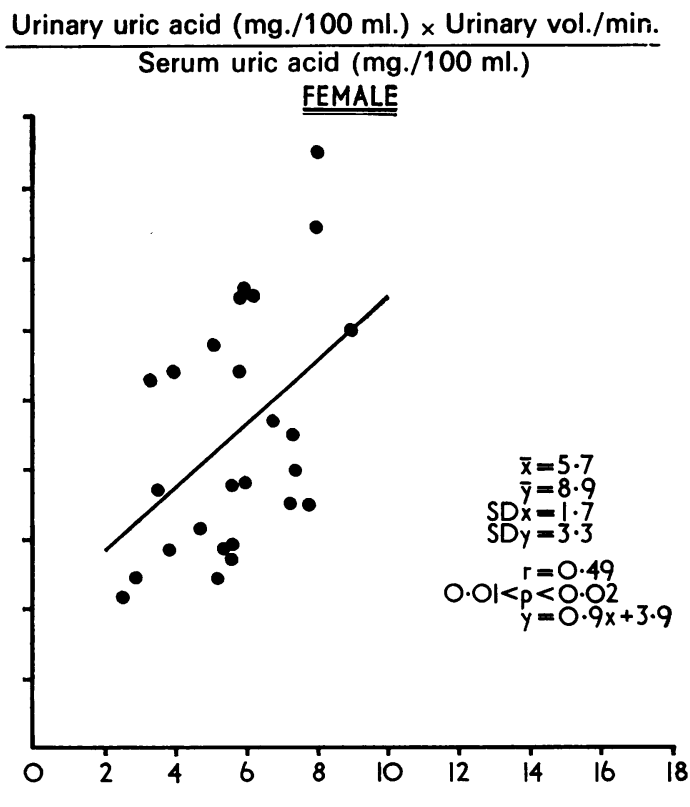

FIG. 5 Uric acid clearance of patients plotted against mean uric acid clearance of male relatives.

Fig. 6 Uric acid clearance of patients plotted against mean uric acid clearance of female relatives 
Mean clearance in gout patients with male relatives was $5.6 \mathrm{ml} . / \mathrm{min}$. (S.D. 1.7) and in those with female relatives $5.7 \mathrm{ml} / \mathrm{min}$. (S.D. 1·7). Mean clearance figures for male and female relatives were $7 \cdot 7 \mathrm{ml}$./min. (S.D. $2 \cdot 2$ ) and $8 \cdot 9 \mathrm{ml} . / \mathrm{min}$. (S.D. 3.3) respectively.

There appears to be a graded correlation between values for patients and those of their relatives. This is more marked in the case of male relatives (Fig. 5: $r=0.77 ; \mathbf{P}<0.001)$. With female relatives there is a wide scatter of clearance values and the correlation is less close (Fig. $6: r=0.49 ; 0.01<P<$ 0.02).

(4) Uric acid: creatinine clearance ratios (Figs 7 and 8)

Similar correlations are found when uric acid clearance is expressed as a ratio to creatinine clearance. The mean ratio $(\times 100)$ in gout patients with male relatives was $5 \cdot 7$, and in those with female relatives $5 \cdot 3$. The mean ratios for male and female relatives were $7 \cdot 5$ and $9 \cdot 1$ respectively.

\section{Discussion}

The higher serum levels of uric acid found in the male relatives is in conformity with studies on normal populations (e.g. Popert and Hewitt, 1962) which show that men tend to have higher levels than women. There was little difference between the mean level of 24-hour urinary uric acid in patients and that of their relatives as a whole, though those of the patients and their male relatives were rather higher than that of female relatives. Many of the relatives had 24-hour urinary uric acid values well above the

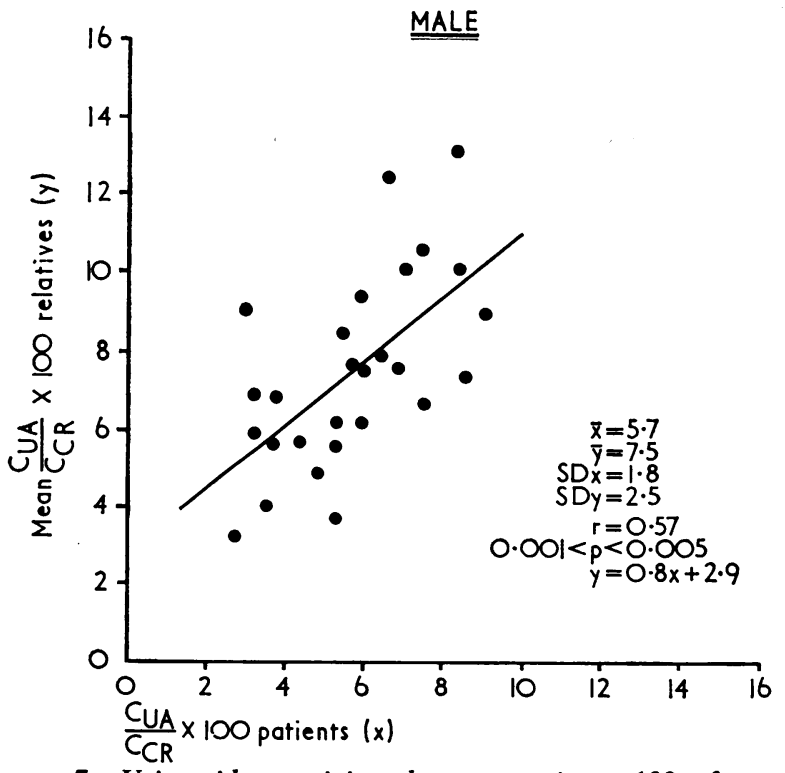

IG. 7 Uric acid: creatinine clearance ratio $\times 100$ of atients compared with the mean ratio of male relatives. accepted upper limit of normal of $600 \mathrm{mg}$. (Seegmiller, Grayzel, Laster, and Liddle, 1961). It is uncertain to what extent their relationship to gouty subjects is responsible for this: the facts that the diet was not entirely free of purines and that uric acid estimation in this study was by Auto Analyzer and not by enzymatic spectrophotometry may have also contributed to the difference. The mean figure for uric acid clearance in the patients is slightly lower than the $7.5( \pm 2.4)$ for gouty men given by Gutman (1964), and the mean figure for male relatives is also slightly lower than his figure for normal men $(8 \cdot 7 \pm 2 \cdot 5)$. The finding of higher clearance figures in female relatives than in male relatives accords with the studies of Wolfson, Hunt, Levine, Guterman, Cohn, Rosenberg, Huddlestun, and Kadota (1949), who found a higher clearance in women than in men.

No correlation could be detected between serum levels of uric acid in patients with gout and the mean levels of their relatives: if such a relationship exists, as it may, it is evident that a much larger number of subjects would have to be studied to demonstrate it. With 24-hour uric acid excretion there appears a tendency for higher values in patients to correlate with mean levels of their relatives, but this is not significant and again a larger study would be necessary to establish the point. With regard to uric acid clearance, however, a significant degree of graded correlation is found between values for patients and mean values for their relatives. This is considerably closer for male relatives than for female relatives, possibly owing to factors arising from the conduct

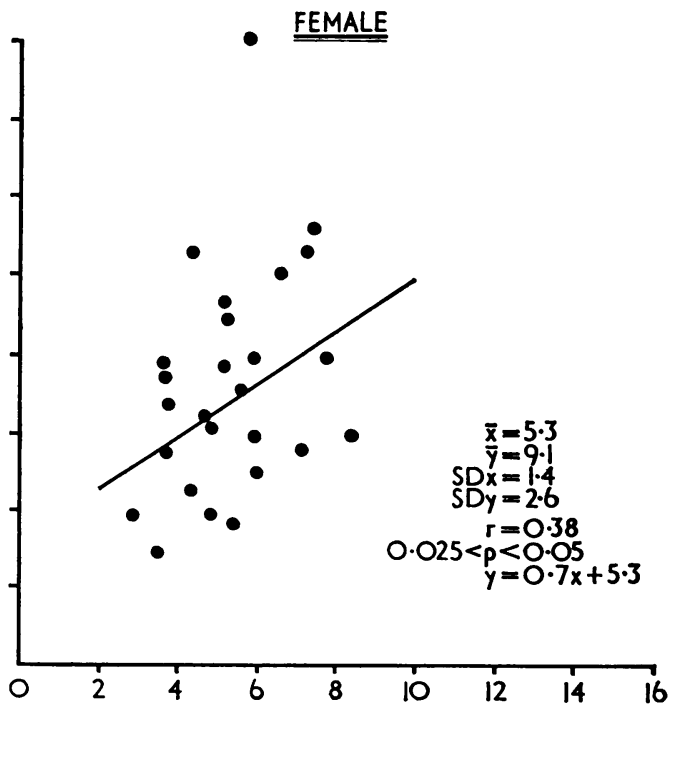

FIG. 8 Uric acid: creatinine clearance ratio $\times 100$ of patients compared with the mean ratio of female relatives. 
of the investigation (e.g. less accurate urine collection in women, although as explained above obviously incomplete collections were excluded) or possibly to a really greater familial aggregation with male relatives.

Strong familial tendencies in gout have teen recognized since early descriptions of the disease (see Talbott, 1967), and hyperuricaemia also appears to be influenced by familial factors. Thus Hauge and Harvald (1955) found serum uric acid levels in the siblings of gouty patients to be significantly higher than those of a control group. Cumulative or multifactorial influences were indicated by the fact that serum uric acid values in the siblings fell into a normal distribution curve, rather than fitting any mendelian pattern, a mode of inheritance which had previously been suggested (Stecker, Hersh, and Solomon, 1949). Further support for the multifactorial concept comes from the increasing number of factors which are known to affect uric acid metabolism and excretion (Scott, 1969). A polygenic control of inheritance is also said to be indicated by the fact that the frequency-distribution curve of serum uric acid in the general population is normal (Finn, Jones, Tweedie, Hall, Dinsdale, and Bourdillon, 1966).

The graded correlation which appears to exist between uric acid clearance values in patients with gout and the mean values of their relatives suggests that the concept of multifactorial influences applying to the level of uric acid in the blood may be extended to the handling of urate by the kidney. If this is accepted, it should nevertheless be emphasized that the data give no information as to the relative importance of genetic as opposed to environmental influences (Kellgren, 1964; Blumberg, 1965), although the effect of a sudden large dietary intake of purine was avoided by the use of a lowpurine diet during the period of study. The eventual answer to the problem will only follow the final identification of the many individual factors regulating uric acid metabolism, but it is likely that hyperuricaemia will in most instances be shown to result from a quantitative variation in a large number of those factors rather than from single major abnormalities.

\section{Summary}

Uric acid clearance was studied in 37 patients with primary gout and 96 of their first-degree relatives. A graded correlation (closer in the case of male relatives than in female relatives) was found between clearance values for patients and mean values for their relatives. It is suggested that the concept of multifactorial influences regulating uric acid levels in the blood can be extended to the renal handling of uric acid.

\section{References}

Blumberg, B. S. (1965) Arthr. and Rheum., 8, 627 (Heredity of gout and hyperuricemia).

Finn, R., Jones, P. O., Tweedie, M. C. K., Hall, S. M., Dinsdale, O. F., and Bourdillon, R. E. (1966) Lancet, 2, 185 (Frequency-distribution curve of uric acid in the general population).

Gutman, A. B., (1964) Amer. J. Med., 37, 833 (Significance of the renal clearance of uric acid in normal and gouty man (Editorial)).

Hauge, M., AND Harvald, B. (1955) Acta med. scand., 152, 247 (Heredity in gout and hyperuricaemia).

Kellgren, J. H. (1964) Ann. rheum. Dis., 23, 109 (The epidemiology of rheumatic diseases).

Popert, A. J., AND HewITT, J. V. (1962) Ibid., 21, 154 (Gout and hyperuricaemia in rural and urban populations).

ScotT, J. T., (1969) Proc. roy. Soc. Med., 62, 851 (Causes of hyperuricaemia).

Seegmiller, J. E., Grayzel, A. I., Laster, L., AND LidDle, L. (1961) J. clin. Invest., 40, 1304 (Uric acid production in gout).

Stecher, R. M., Hersh, A. H., AND Solomon, W. M. (1949) Ann. intern. Med., 31, 595 (The heredity of gout and its relationship to familial hyperuricaemia).

TAlbotT, J. H. (1967) 'Gout', 3rd ed., p. 13. Grune and Stratton, New York and London.

Wolfson, W. Q., Hunt, H. D., Levine, R., Guterman, H. S., Cohn, C., RosenberG, E. F., Huddlestun, B., AND KADOTA, K. (1949) J. clin. Endocr., 9, 749 (The transport and excretion of uric acid in Man. V. A sex difference in urate metabolism). 